Psychother Psychosom 2012;81:315-317

DOI: $10.1159 / 000336811$

\title{
Fish Oil for Attenuating Posttraumatic Stress Symptoms among Rescue Workers after the Great East Japan Earthquake: A Randomized Controlled Trial
}

Daisuke Nishia-d, Yuichi Koido ${ }^{\text {b, e }}$, Naoki Nakaya ${ }^{f}$, Toshimasa Sone $^{\mathrm{g}}$, Hiroko Noguchic, Kei Hamazakic, h, Tomohito Hamazakic, i, Yutaka Matsuoka ${ }^{\mathrm{a}-\mathrm{d}, \mathrm{j}}$

${ }^{a}$ Department of Psychiatry and ${ }^{b}$ Clinical Research Institute, National Disaster Medical Center, ${ }^{C}$ CREST, Japan Science and Technology Agency, ${ }^{d}$ National Institute of Mental Health, National Center of Neurology and Psychiatry, and ${ }^{\mathrm{e}} \mathrm{Head}$ Office, Japan Disaster Medical Assistance Team, Tokyo; fDepartment of Nutrition and Dietetics, Faculty of Family and Consumer Science, Kamakura Women's University, Kamakura; ${ }^{9}$ Department of Rehabilitation, Faculty of Health Science, Tohoku Fukushi University, Sendai; h Department of Public Health, Faculty of Medicine, and 'Department of Clinical Sciences, Institute of Natural Medicine, University of Toyama,

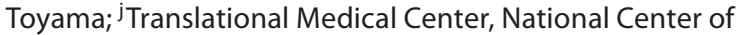
Neurology and Psychiatry, Tokyo, Japan

The Great East Japan Earthquake and tsunami that occurred on March 11, 2011 devastated the northeastern coast of Japan, and left about 20,000 dead or missing. Many rescue workers, as well as survivors, were exposed to traumatic experiences. An appropriate strategy for the attenuation of posttraumatic stress disorder (PTSD) symptoms is therefore clearly required.

In the pathogenesis of PTSD, fear memory becomes excessively consolidated and extinction learning does not progress [1]. Kitamura et al. [2] indicated that the level of hippocampal neurogenesis is a crucial factor in determining the period of hippocampal-dependent fear memory. This finding suggested that the fear memory, which is a characteristic of PTSD, might be controlled by appropriately regulating hippocampal neurogenesis [3].

Based on the animal research conducted to date, fish oil is the most promising candidate for dietary intervention to facilitate adult hippocampal neurogenesis [4]. In an open-label trial in patients with physical injury, we previously found that PTSD symptoms at 12 weeks after injury were significantly alleviated by taking fish oil containing mainly docosahexaenoic acid (DHA) [5]. Against this background, this study aimed to determine whether fish oil supplementation can attenuate PTSD symptoms among Disaster Medical Assistance Team members who were deployed during the acute disaster phase of the Great East Japan Earth-

\section{KARGER}

E-Mail karger@karger.ch www.karger.com/pps
(C) 2012 S. Karger AG, Basel $0033-3190 / 12 / 0815-0315 \$ 38.00 / 0$

Karger Open access

This is an Open Access article licensed under the terms of the Creative Commons Attribution- NonCommercial-NoDerivs 3.0 License (www.karger.com/OA-license), applicable to the online version of the article only. Distribution for non-commercial purposes only. quake. Disaster Medical Assistance Team members are doctors, nurses, and operational coordination staff (medical or clerical staff who are neither doctors nor nurses) who are dispatched as a mobile medical team with specialized training that is capable of acting during the acute phase of a large-scale disaster.

This single-blind, randomized, parallel-group trial was approved by the Ethics Committee of the National Disaster Medical Center, Tokyo, Japan, on April 1, 2011, and registered at UMIN Clinical Trials Registry as UMIN 000005367. The detailed trial procedures and baseline assessment have been reported elsewhere [6].

Participants provided informed consent and were randomly assigned to either the fish oil supplementation plus psychoeducation group or the psychoeducation alone group. For participants allocated to the fish oil plus psychoeducation group, 7 capsules per day, each containing $320 \mathrm{mg}$ of fish oil, were provided in line with previous research [7]. The fish oil composition of each capsule was 70\% DHA and 7\% eicosapentaenoic acid (EPA). For participants of both groups, a leaflet on psychoeducation about posttraumatic distress focusing on critical incident stress was provided.

The primary outcome was a total score on the Impact of Event Scale-Revised (IES-R) at 12 weeks after shipment of the supplements on April 19, 2011. The IES-R is a self-reporting questionnaire about PTSD symptoms and is the most widely used measure in all forms of disaster area research [8]. Safety of the intervention was evaluated by the presence of adverse events during the observation period, by asking the participants about the presence of such events at 2, 4, 8 , and 12 weeks after the start of fish oil supplementation.

All analyses were conducted according to the intention-totreat principle. Analysis of covariance was used to investigate the significance of the differences in the initial values as well as of the net changes after the intervention among the 2 groups. Covariates for the analysis of covariance were sex, age, and IES-R score at baseline. We examined the impact of sex difference for fish oil supplementation on PTSD symptoms as scheduled, because previous studies showed that the prevalence of PTSD was higher in women than in men [9]. All statistical analyses used two-tailed tests. For all statistical evaluations, $\mathrm{p}$ values less than 0.05 were considered indicative of significant differences. All data analyses were performed using SAS version 9.1 (SAS Institute Inc., Cary, N.C., USA).

Of the 1,816 Disaster Medical Assistance Team workers deployed to the disaster areas, 172 participated in the study between April 2 and 12,2011. The participants did not differ significantly from nonparticipants in terms of occupation. The two groups were well balanced with respect to baseline characteristics. Primary outcome data were available for all participants except one. A sensitivity analysis was performed using a multiple imputation
Yutaka Matsuoka, MD, $\mathrm{PhD}$

Department of Psychiatry, National Disaster Medical Center 3256 Midoricho, Tachikawa

Tokyo 190-0014 (Japan)

Tel. +81 42526 5511, E-Mail yutaka @ ncnp.go.jp 
Table 1. Change in IES-R scores of participants in the intervention and control groups stratified by sex

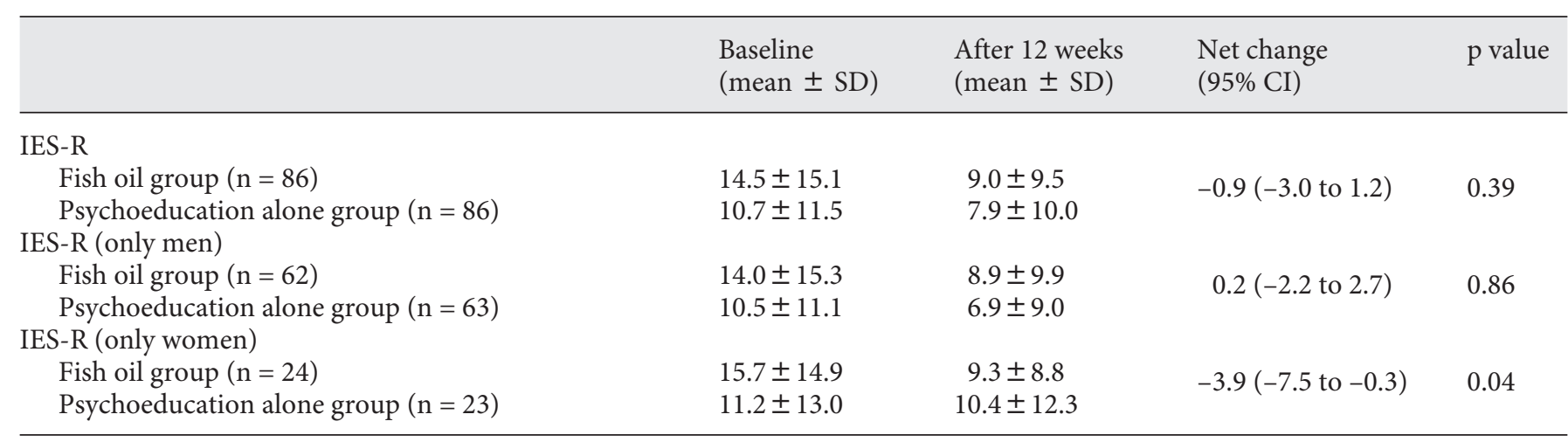

Net change: analysis of covariance adjusted for age and IES-R score at baseline.

procedure to impute an IES-R score at the follow-up assessment for 1 participant who dropped out. The mean duration from baseline assessment to follow-up was 14.2 weeks $(\mathrm{SD}=0.9)$, and from shipment of the supplements to follow-up assessment it was 12.6 weeks $(\mathrm{SD}=0.8)$.

When adjusted for age, sex, and IES-R score at baseline, there was no significant difference in change of IES-R scores between the two groups. However, in women, there was a significant difference between the two groups when adjusted for age and IES-R scores at baseline (table 1). In terms of safety, the occurrence rate of adverse events was not significantly different between the two groups, and none were regarded as serious or led to withdrawal.

Unfortunately, the trial did not show the superiority of fish oil supplementation plus psychoeducation over psychoeducation alone for attenuating PTSD symptoms. It is unclear why fish oils play an important role in regulating psychological well-being only in women. Future studies should determine the effect of the sex difference.

Based on previous studies [4], DHA appeared to facilitate adult hippocampal neurogenesis. However, there is evidence to support the effectiveness of EPA monotherapy or a combination of EPA and DHA for depressive disorders [10]. Because no previous studies have examined the effectiveness of fish oils to attenuate PTSD symptoms, the appropriate composition of fish oil capsules warrants attention.

The study has some limitations. First, the study was not a placebo-controlled, double-blind trial. Because this study was implemented at a time of crisis, we could not prepare placebo capsules. It might be possible that we found a placebo effect that acted more strongly in women who took the fish oil supplements. Second, this study relied on self-reports of adherence to the protocol, rather than biomarkers. Third, many rescue workers invited to participate did not respond, which limits the external validity of the findings.

In conclusion, fish oil supplementation may offer a safe strategy for attenuating PTSD in women, and thus is an important topic that should be further explored in disaster mental health care.

\section{Acknowledgments}

The authors would like to thank Prof. Kaoru Inokuchi for his generous financial support. They also thank Dr. Hisayoshi Kondo and Mr. Masayuki Ichihara for coordination with participants, Dr. Masato Usuki for his generous support, and Mss. Kyoko Akutsu and Yumiko Kamoshida for data management and Ms. Hiroko Hamatani for preparation of bottled supplements. Prof. Yasuhiro Otomo, Prof. Takeshi Terao and Dr. Katsumi Ikeshita joined this study as members of the data and safety monitoring board. All of the supplements used in the study were supplied by Kentech Co., Ltd., Toyama, Japan. This work was supported by CREST, the Japan Science and Technology Agency. The Japan Science and Technology Agency had no role in the study design, data collection and analysis, decision to publish, or preparation of the manuscript.

\section{Disclosure Statement}

Dr. Nishi has received research support from Toray Industries, Inc. and the Foundation for Total Health Promotion, and lecture fee from Qol Co., Ltd. and DHA and EPA Association. Dr. Koido has received research support from the Ministry of Health, Labor, and Welfare of Japan, Ono Pharmaceutical Co., Ltd., Astrazeneca K.K., Bristol-Myers Squibb Company, and National Center of Global Health and Medicine. Dr. K. Hamazaki has received research support from the Japan Society for the Promotion of Science, the Tamura Foundation for Promotion of Science and Technology, and the Ichiro Kanehara Foundation for Promotion of Medical Sciences and Medical Care, and consultant fees from Polyene Project, Inc. and Otsuka Pharmaceutical Co., Ltd., and lecture fee from Nippon Suisan Kaisha, Ltd. Dr. T. Hamazaki has received research support from the Japan Society for the Promotion of Science, Open Research Center for Lipid Nutrition (Kinjo Gakuin University), and Nippon Suisan Kaisha, Ltd., and consultant fees from Polyene Project, Inc. and Otsuka Pharmaceutical Co., Ltd., and lecture fees from Mochida Pharmaceutical Co., Ltd. Dr. Matsuoka has received research support from the Japan Science and Technology Agency, CREST and the Ministry of Health, Labor, and Welfare of Japan, and lecture fee from Eli Lilly Japan KK and Otsuka Pharmaceutical Co., Ltd. All other authors declare that they have no competing interests. 


\section{References}

1 Ressler KJ, Mayberg HS: Targeting abnormal neural circuits in mood and anxiety disorders: from the laboratory to the clinic. Nat Neurosci 2007;10:1116-1124.

2 Kitamura T, Saitoh Y, Takashima N, Murayama A, Niibori Y, Ageta H, Sekiguchi M, Sugiyama H, Inokuchi K: Adult neurogenesis modulates the hippocampus-dependent period of associative fear memory. Cell 2009;139:814-827.

3 Matsuoka Y: Clearance of fear memory from the hippocampus through neurogenesis by omega- 3 fatty acids: a novel preventive strategy for posttraumatic stress disorder? Biopsychosoc Med 2011;5:3.

-4 Kawakita E, Hashimoto M, Shido O: Docosahexaenoic acid promotes neurogenesis in vitro and in vivo. Neuroscience 2006;139:991-997.

$\checkmark 5$ Matsuoka Y, Nishi D, Yonemoto N, Hamazaki K, Hashimoto K, Hamazaki T: Omega-3 fatty acids for secondary prevention of posttraumatic stress disorder after accidental injury: an open-label pilot study. J Clin Psychopharmacol 2010;30:217-219.
6 Matsuoka Y, Nishi D, Nakaya N, Sone T, Hamazaki K, Hamazaki T, Koido Y: Attenuating posttraumatic distress with omega-3 polyunsaturated fatty acids among disaster medical assistance team members after the Great East Japan Earthquake: the APOP randomized controlled trial. BMC Psychiatry 2011;11:132.

7 Hamazaki T, Sawazaki S, Itomura M, Asaoka E, Nagao Y, Nishimura N, Yazawa K, Kuwamori T, Kobayashi M: The effect of docosahexaenoic acid on aggression in young adults. A placebo-controlled doubleblind study. J Clin Invest 1996;97:1129-1133.

8 Weiss DS: The Impact of Event Scale-Revised, ed 2. New York, Guilford Press, 2004.

-9 Kessler RC, Sonnega A, Bromet E, Hughes M, Nelson CB: Posttraumatic stress disorder in the National Comorbidity Survey. Arch Gen Psychiatry 1995;52:1048-1060.

10 Sublette ME, Ellis SP, Geant AL, Mann JJ: Meta-analysis of the effects of eicosapentaenoic acid (EPA) in clinical trials in depression. J Clin Psychiatry 2011;72:1577-1584. 